of glycine which achieve similar degrees of inhibition after electrophoretic or synaptic release. If this is so, the results obtained in the goldfish ${ }^{1}$ provide no evidence for a presynaptic action of strychnine, and support a trans. mitter role of glycine, or a "glycine-like" amino-acid $2-4$, at vertebrate strychnine-sensitive inhibitory synapses.

D. R. CURTIS

G. A. R. Johnston

Department of Physiology,

Australian National University,

Canberra, Australia.

Received September 29, 1969.

${ }^{1}$ Roper, S., Diamond, J., and Yasargil, G. M., Natme, 223, 1168 (1969).

${ }^{2}$ Curtis, D. R., Hösli, L., Johnston, G. A. R., and Johnston, I. H., Frrp. Brain Res., $5,235(1068)$.

${ }^{3}$ Curtis, D. R., Hösli, L., and Johnston, G. A. R., Exp. Erain Res., 6, 1 (1968).

4 Curtis, D. R., Duggan, A. W., and Johnston, G. A. R., Brain Res., 14, 759 (1969).

${ }^{3}$ Curtis, D. R., and Ryall, R. W., Exp. Brain Res., 2, 81 (1.966).

\section{Strychnine Antagonism and Glycine: a Reply}

Drs Curtis and Johnston seem to have overlooked certain items in our experiments. The important question is, should one expect the concentration of strychnine which blocks the action of a synaptically relcased transmitter to cause a reduction in the action of the same substance delivered iontophoretically to the neurone? Our critics quote experiments in which they measured the inhibitory hyporpolarization, which is of course a function of the driving force on the ions, as well as the change in mcmbrane resistance. In our experiments these facts must be stressed: (a) 'The physiological response we measured, the increased membrane conductance (a direct measure of transmitter action), was largely the rosult of synaptic action on the region of the cell from which we recorded, and the glycine was pipctted onto the same region of the cell (and indeed must sometimes have rcached even more distant regions also). The contribution to our recorded. conductance changes of physiological inhibitory activity more than about $200 \mathrm{\mu m}$ along the dendrites would be small. We did not detail the evidence for these items, but simply quoted the work on which they are based ${ }^{1}$. (b) The strychnine concentration about the cell, as detected by the blocking of physiological inhibition, rose gradually until the effects of two independently activated inputs were totally prevented (wo would have detected an inhibition as little as 2 per cent of the control). (c) At this critical blocking concentration of strychnine, glycinc effects were unchanged over a range of doses from the just detectable to the obviously "saturating" (not only doses "which achieve similar degrees of inhibition after electrophoretic or synaptic release"). The smallest glycine dose whose effect wo could reliably measure caused a $1-2$ per cont inhibitory conductance change; this was less than $1 / 20$ of that caused by a "saturating" dose. "These small responses to gly cine should be compared with the relatively enormous conductance change during synaptic activation (50-60 per cent incrcase). It is difficult to imagine that this threshold glycine dose was causing a "more intense activation of a smaller area of the postsynaptic membrane" than that involved in the full physiological responsc. Nevertholess, the direct offects of this small dose of glycine (and of the larger doses) were quantitatively unaffected, even though the very large physiological responso was not just reduced but totally eliminated.

The point we wish to make is that experiments which attempt to answer the questions we asked are necessary in order to exclude the possibilities raiscd in our report. If the blocking action of strychnine is to be used as evidence, then the investigation must be made quantitatively, with regard to the critical concentration of strychnine, the dose-response relations of glycine, and the extent of cell activated both physiologically and pharmacologically. It is important to note the results of the analogous experiment performed at the neuromuscular junction: curare, in uniform concentration over the preparation, was somewhat more effective in reducing the end-plate potential than in reducing the iontophoretically applied acetylcholine potential, but the effects of small doses of acetylcholine seemed to be readily abolished ${ }^{2}$. These findings further strengthen our concern about accopting the qualitative observation that strychnine blocks both physiological and pharmacological inhibition as evidence for the identification of the transmitter. Our results still leave the possibility that a glycine-like substance (or perhaps a glycine complex) is a transmitter. But the postsynaptic action of strychnine, as revealed by glycine itself, seems to require larger concentrations of strychnine than are necessary to block tho effects of physiological inhibition (at least on the Mauthner cell).

S. ROPER

J. DIAMOND

Department of Physiology,

University College,

London WC1.

Received December 4, 1969; revised Jamumy 9, 1970.

1 Diamond, J., J. Physiol., 194, 669 (1968).

${ }^{2}$ Goldsmith, T. H., J. Physiol., 165, 368 (1963).

\section{5-Hydroxyindoleacetic Acid Levels in the Cerebrospinal Fluid of Depressive Patients treated with Probenecid}

ACCORDING to the serotonin (5-hydroxytryptamine, 5-HT) hypothesis there is a causal relationship between mental depression and 5-HT deficiency in the brain ${ }^{1-3}$. Some depressive patients - mainly those suffering from an endogenous depression-display the following clinical signs. (1) The concentration of 5-hydroxyindoleacetic acid (5-HIAA) in the cerebrospinal fluid (CSF) (refs. 4 and 5) and the urinary concentrations of 5-HT (ref. 6) and 5-HIAA (refs. 7 and 8) are unusually low. (2) The transformation of 5-hydroxytryptophan into 5-HT seems to be defective $^{9}$ (although in a later publication Coppen ${ }^{1}$ reported his inability to reproduce those findings). (3) Precursors of $5 \cdot \mathrm{HT}$ which readily enter the brain-5hydroxytryptophan ${ }^{10}$ and truptophan ${ }^{11}$ can have a therapeutic effect. Finally, in suicide victims the cerebral concentration of indoleamines proved to differ from that in a controi group and, according to Shaw et al. ${ }^{12}$, the 5-HT concentrations were diminished. Bourne et al. ${ }^{13}$ were unable to corroborate this but did find a diminished concentration of 5 -HIAA.

These data pertain to absolute concentrations and offer insufficient information on the rate of metabolism of 5 -HT. It is evident that suitable methods for tho calculation of the turnover of $5-\mathrm{HT}$ in the human brain are required before the serotonin hypothesis can be established. Neff et al. ${ }^{14}$ have found, in rats, that the transport of 5-HIAA from the brain into the bloodstream is almost totally inhibited by $p$-(dipropylsulphamoyl)benzoic acid (probenecid). They also suggested that the rate of accumulation of 5-HTAA under the influence of probenecid could be equated with the rate of synthesis of 5 -HT in the brain. In dogs, the concentration of 5-HIAA in the CSF increased following probenecid administration ${ }^{15}$. There aro indications (albeit indirect) that acid amine metabolites in CSF are derived from brain amines and that changes in the CSF acid concentrations may reflect the metabolism of the cerebral amine precursor ${ }^{16,17}$. If this is true, then the rate of increaso of the 5-HLAA concentration in the CSF 